\title{
Long-Term Favorable Course of Aspergillus Endo-, Myo-, and Pericarditis
}

\author{
Akiko Hayashi, ${ }^{1}$ MD, Sayuki Kobayashi, ${ }^{1}$ MD, Itaru Hisauchi, ${ }^{1}$ MD, Takaaki Komatsu, ${ }^{1}$ MD, \\ Shiro Nakahara, ${ }^{1}$ MD, Yoshihiko Sakai, ${ }^{1}$ MD, Kosuke Haruki, ${ }^{2}$ MD and Isao Taguchi, ${ }^{1}$ MD
}

\begin{abstract}
Summary
Here, we report on a healthy 30-year-old man with no significant medical history, who tested negative for human immunodeficiency virus antigenemia but developed Aspergillus pancarditis. A case of this kind is extremely rare, and to the best of our knowledge, this is the first report of a patient with Aspergillus pancarditis, which generally leads to a very poor outcome, who had a long-term favorable clinical course. A biopsy from the right atrium of hypertrophied atrial septum was essential for obtaining the definitive diagnosis. Long-term administration of an effective antifungal oral agent might account for the patient's favorable outcome.
\end{abstract}

(Int Heart J 2017; 58: 1020-1023)

Key words: Pancarditis, Biopsy

A spergillus species infections can be severe and invasive, involving almost every major organ system. Aspergillus pancarditis is especially rare, with a very poor prognosis. Our patient with Aspergillus pancarditis had a long-term favorable course, which might be accounted for by the following important factors: a definitive diagnosis by a biopsy from the right atrium, early administration of an antifungal agent, timely changing to a more effective agent, and continuation of treatment based on serum $\beta$-d-glucan levels. This report should provide helpful information for the treatment of similar cases.

\section{Case Report}

We report a 30-year-old male patient with no significant medical, family, or occupational history, who had never used any intravenous drugs. The chief complaints of high fever and cough appeared in the latter third of March 2013; he was admitted to another hospital in April of the same year and received antibiotic therapy. However, his fever of $38^{\circ} \mathrm{C}$ and cough persisted, and he was admitted to our hospital for specialized workup and treatment.

Malignant lymphoma or infective endocarditis was suspected at the time of his admission, based on test results indicating inflammation (C-reactive protein $=4.2$ $\mathrm{mg} / \mathrm{mL}$, reference range $<0.3 \mathrm{mg} / \mathrm{mL}$ ), chest computed tomography (CT) imaging showing pericardial effusion without findings suggestive of pulmonary aspergillosis, echocardiogram showing vegetations (Figure 1A and B), and gallium scintigraphy scan showing abnormal accumulation of gallium within the pericardial space. However, because of an abnormally high serum $\beta$-d-glucan level $(612 \mathrm{pg} / \mathrm{mL}$, reference range $<20 \mathrm{pg} / \mathrm{mL})$, which suggested fungal infection, we started an intravenous antifungal agent (voriconazole $400 \mathrm{mg} /$ day). Transesophageal echocardiography showed extensive thickening of both atrial walls, focused around the mitral annular ring, and including the atrial septum (Figure 2). Moreover, malignant lymphoma was suspected because of the large pericardial effusion, and pericardiocentesis was performed. Approximately $800 \mathrm{~mL}$ of yellow to faintly bloody pericardial fluid was obtained. The cytological diagnosis was negative for malignancy; therefore, we performed a myocardial biopsy. Under intracardiac ultrasound guidance, we obtained a sample of the hypertrophied atrial septum from the right atrium, near the oval window. The histopathological findings included scattered macrophages, lymphocytes, and eosinophils. Elongated figures scattered among the cells appeared to be fungal filaments (Figure 3 A). Grocott staining revealed the same filamentous structures (Figure 3B), and blood tests were positive for the Aspergillus antigen $(2.8 \mathrm{ng} / \mathrm{mL}$, reference range $<0.5 \mathrm{ng} /$ $\mathrm{mL}$ ). Aspergillus pancarditis (endocarditis, myocarditis, and pericarditis) was diagnosed, and the antifungal agent was continued. However, because the abnormally elevated serum $\beta$-d-glucan levels persisted $(1180 \mathrm{pg} / \mathrm{mL})$, the patient's treatment was changed on day 37 from voriconazole $400 \mathrm{mg} /$ day to amphotericin B $150 \mathrm{mg} /$ day. Beginning around day 50, the $\beta$-d-glucan level started to decrease. Echocardiography findings indicated improvement of the mural hypertrophy by day 50, and the mobile, vegetation-like shadow attached to the mitral annular ring

From the ${ }^{1}$ Department of Cardiology, Dokkyo Medical University Koshigaya Hospital and ${ }^{2}$ Department of Infection Control, Dokkyo Medical University Koshigaya Hospital, Saitama, Japan.

Address for correspondence: Isao Taguchi, MD, Department of Cardiology, Dokkyo Medical University Koshigaya Hospital, 2-1-50 Minami-Koshigaya, Koshigaya, Saitama 343-8555, Japan. E-mail: taguchi@ dokkyomed.ac.jp

Received for publication September 27, 2016. Revised and accepted January 4, 2017.

Released in advance online on J-STAGE November 22, 2017.

doi: 10.1536/ihj.16-485

All rights reserved by the International Heart Journal Association. 

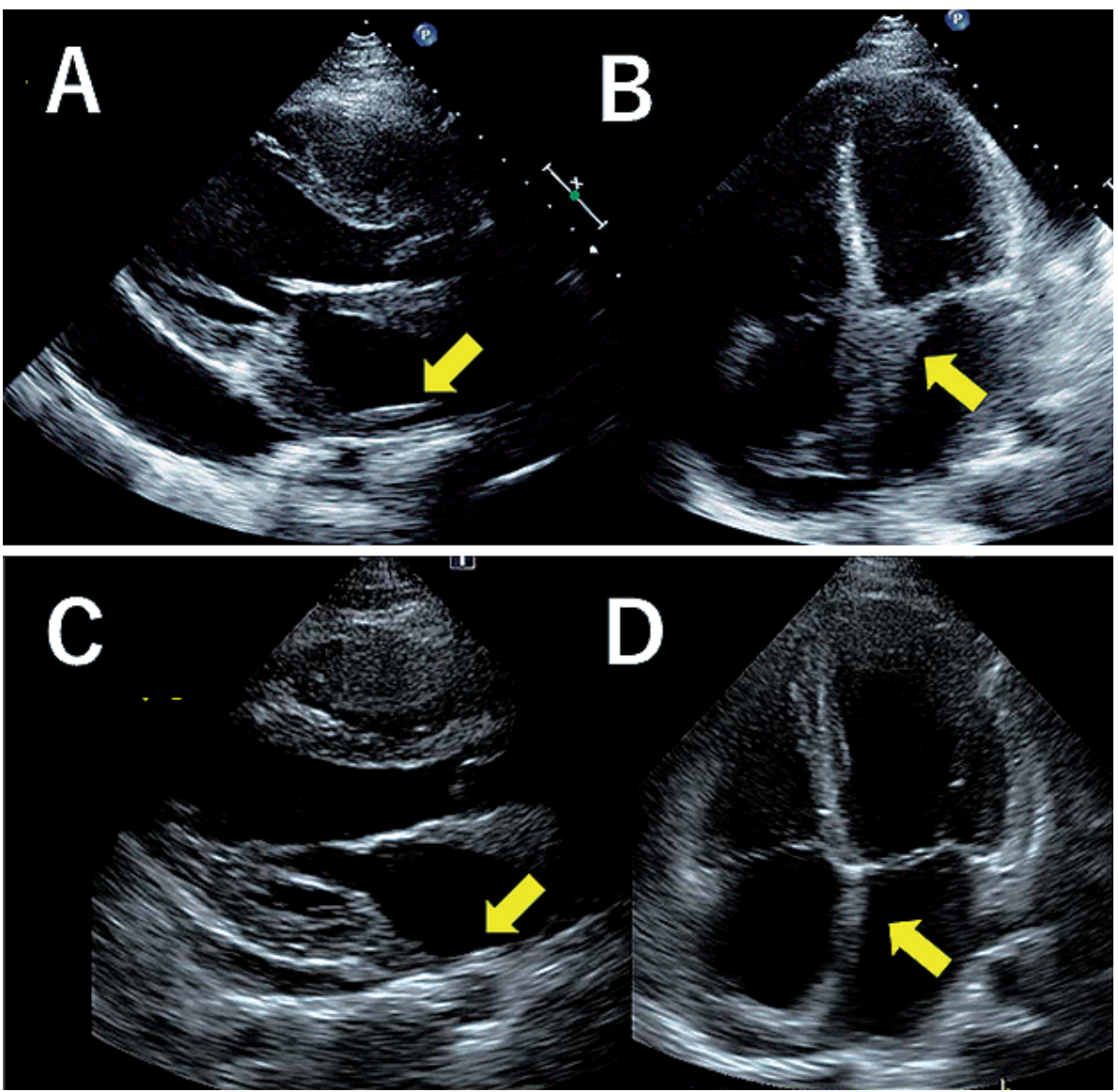

Figure 1. Transthoracic echocardiography images obtained before treatment in $\mathbf{A}$ and $\mathbf{B}$ and after treatment in $\mathbf{C}$ and $\mathbf{D}$. Vegetations are seen in $\mathbf{A}$ and $\mathbf{B}$ (yellow arrows).

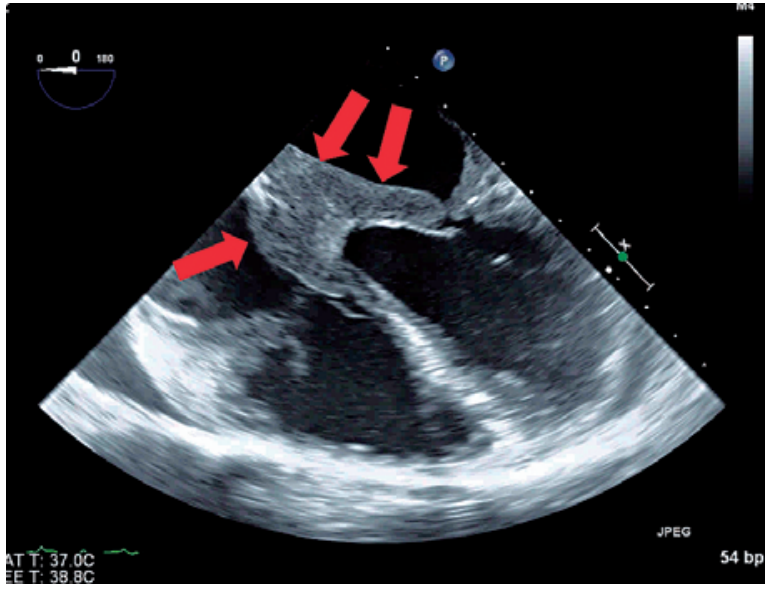

Figure 2. Transesophageal echocardiogram at the time of admission. Marked hypertrophy of both atrial walls from the mitral annular ring to the atrial septum is seen (red arrows).

had also disappeared. Three months after admission, hypertrophy affecting the base of the aorta; the walls of both atria, including the atrial septum; and the base of the posterior atrial wall had further improved to almost normal (Figure 1C and D). Furthermore, the pericardial effusion did not return after pericardiocentesis.

The electrocardiogram showed atrial flutter with 5:1 conduction and a heart rate (HR) of 40 beats per minute (bpm) from the time of admission (Figure 4A) and junctional rhythm $(\mathrm{HR}=48 \mathrm{bpm})$ from day 50 (Figure $4 \mathrm{~B}$ ). Three months after admission, the patient's sinus rhythm returned $(\mathrm{HR}=70 \mathrm{bpm}$; Figure $4 \mathrm{C})$.

Renal dysfunction manifesting as a high serum creatinine level $(1.5 \mathrm{mg} / \mathrm{dL}$, reference range $<1.0 \mathrm{mg} / \mathrm{dL})$ was observed from around day 60 , and because nephrotoxicity is associated with amphotericin B, the patient's therapy was changed to oral voriconazole $400 \mathrm{mg} /$ day. The patient's renal function did not deteriorate further, and his serum creatinine level decreased to $1.2 \mathrm{mg} / \mathrm{dL}$.

Other laboratory data, including complete blood count, parameters of liver and renal function, and creatinine kinase levels, did not change markedly over the course of treatment. The changes in test values during the patient's hospitalization are as follows: white blood cell counts $=4.9-8.6 \times 10^{3} / \mu \mathrm{L}$ (reference range: $4.0-9.0 \times 10^{3} /$ $\mu \mathrm{L}$ ), hemoglobin levels $=12.6-15.2 \mathrm{~g} / \mathrm{dL}$ (reference range: $12.0-18.0 \mathrm{~g} / \mathrm{dL})$, platelet counts $=17.2-24.8 \times 10^{4} / \mu \mathrm{L}($ reference range: $\left.17.0-42.0 \times 10^{4} / \mu \mathrm{L}\right)$, glutamic-oxaloacetic transaminase levels $=21-40 \mathrm{U} / \mathrm{L}$ (reference range: $0-44 \mathrm{U} /$ L), glutamic-pyruvic transaminase levels $=13-27 \mathrm{U} / \mathrm{L}$ (reference range: 0-47 U/L), creatinine kinase levels $=56$ - 

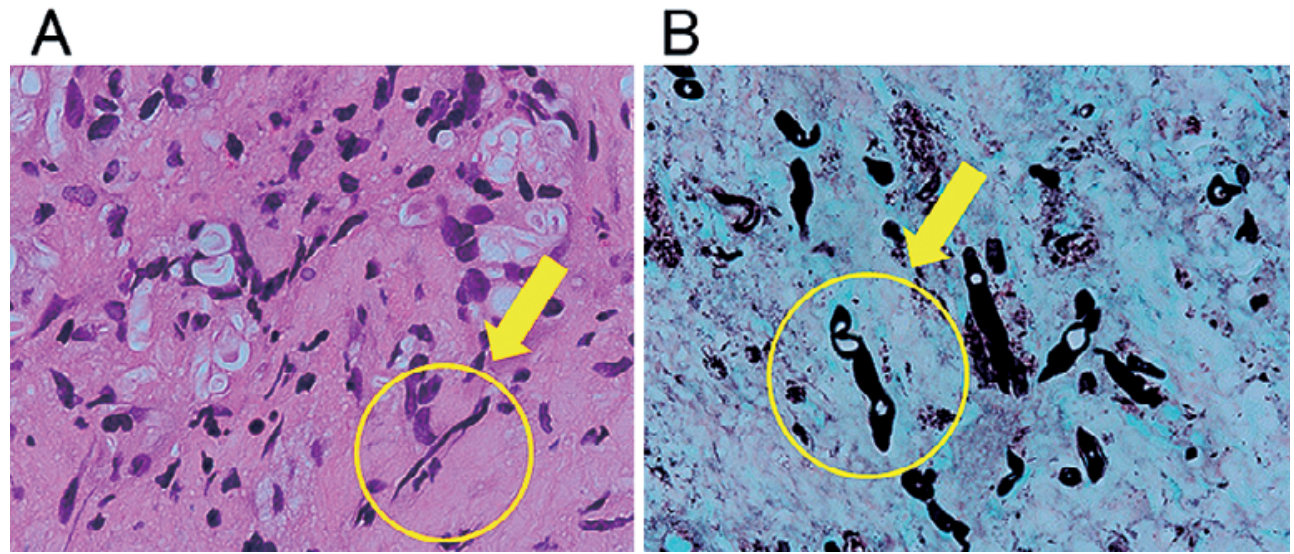

Figure 3. Pathology findings. A: HE stain $\times 1,000$. Scattered macrophages, lymphocytes, eosinophils. Elongated shapes between the cells appear to be fungal filaments (yellow arrows). B: Grocott stain $\times 1,000$. The filaments are short, friable, and swollen, suggestive of filamentous fungi (yellow arrows).

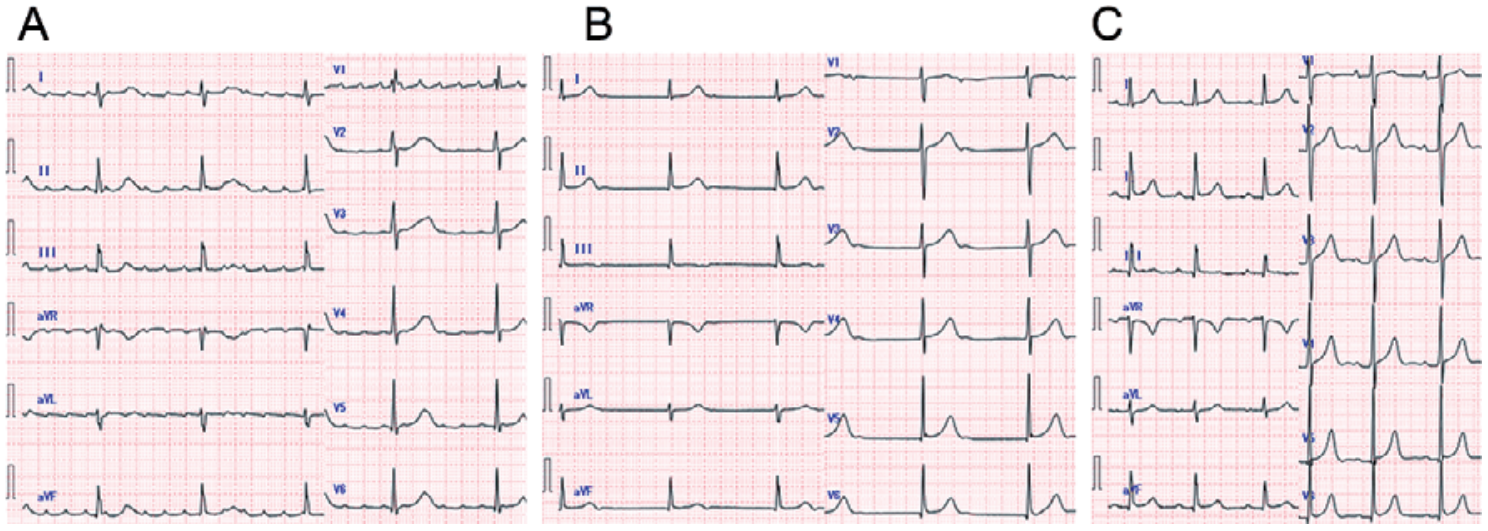

Figure 4. ECG changes. A: admission, B: day 50, C: 3 months after admission.

$65 \mathrm{U} / \mathrm{L}$ (reference range: 57-174 U/L), creatinine kinase$\mathrm{MB}$ isozyme levels $=3.2-3.4 \mathrm{U} / \mathrm{L}$ (reference range: $0-12$ $\mathrm{U} / \mathrm{L}$ ). The patient's body temperature improved to $36.5^{\circ} \mathrm{C}$ at day 10 after admission and remained below $37^{\circ} \mathrm{C}$.

Although the patient was restarted on oral voriconazole at around day 60 because of amphotericin Bassociated nephrotoxicity, his Aspergillus-related symptoms were not exacerbated, and his $\beta$-d-glucan values decreased to $144 \mathrm{pg} / \mathrm{mL}$. Subsequent Aspergillus antigen testing was negative, and the patient was discharged on day 148 with a prescription for ongoing oral voriconazole.

\section{Discussion}

Aspergillus infection usually affects the lungs, with rapid hematogenous dissemination to all organs and formation of microabscesses. ${ }^{1)}$ When Aspergillus infects the heart, it causes an acute pathological state, including endocarditis, myocarditis, and pericarditis. ${ }^{2)}$ Symptoms progress insidiously and rapidly during myocarditis, and patients commonly die within a few hours to a few days. ${ }^{3,4)}$ We believe that the favorable clinical outcome of our patient was accounted for by the patient's normal left ventricular function, the absence of an underlying disease that would cause impaired immunity, and the effectiveness of the antifungal agent against our patient's infection. Furthermore, the finding that creatinine kinase levels, including creatinine kinase-MB isozyme, were within the normal range, suggesting that the patient's Aspergillus infection might have been localized to the atria and conduction system; the localized infection might have been one of the most important factors leading to the patient's favorable outcome.

Identifying the route of infection was a challenge in this case. Usually the pathogen reaches the lungs through inhalation; however, there was no lesion suggesting respiratory infection on the CT images of our patient. There was no history of penetrating trauma. There are various routes of infection, but usually Aspergillus does not spread hematogenously unless the patient has impaired immunity. Our patient was a healthy young man with no significant medical history, who was negative for human immunodeficiency virus antigenemia.

The definitive diagnosis of Aspergillus infection by standard sputum cultures is difficult, and tissue biopsy is usually required. This invasive test provides the correct diagnosis in $10 \%-30 \%$ of cases. The blood culture positivity rate is extremely low, at $6.4 \%-11 \% .^{5)}$ Aspergillus was not 
isolated from our patient's blood or pericardial fluid cultures but was histopathologically identified from myocardial tissue. The biopsy sample from the right atrium of hypertrophied atrial septum was essential for obtaining the definitive diagnosis in our patient.

Treatment for Aspergillus carditis is based on antifungal agents such as amphotericin B. However, systemic infection usually occurs during the time of diagnosis, and because the infection rapidly progresses, the prognosis is extremely poor. However, this case represents a favorable clinical course; more than 2 years have elapsed since the patient's treatment was changed to oral voriconazole. Data on the duration of oral antifungal administration during maintenance therapy are scarce, and to date, we have continued administration of voriconazole. This case is important, because to the best of our knowledge, this is the first report of Aspergillus pancarditis with a long-term favorable clinical course. Going forward, we will also need to consider the risk of reactivation of infection, and whether or not diligent follow-up observations, including echocardiography, ECG, and sample collection and testing are needed.

\section{Disclosures}

Conflicts of interest: None of the authors have any financial or other interest in the product or distributor of the product.

\section{References}

1. Rinaldi MG. Invasive aspergillosis. Rev Infect Dis 1983; 35: 1061-77. (Review).

2. Atkinson JB, Connor HA, Robinowitz M, McAllister HA, Virmani R. Cardiac fungal infections: review of autopsy findings in 60 patients. Hum Pathol 1984; 15: 935-42.

3. Schønheyder H, Hoffmann S, Jensen HE, Hansen BF, Franzmann MB. Aspergillus fumigatus fungaemia and myocarditis in a patient with acquired immunodeficiency syndrome. APMIS 1992; 100: 605-8.

4. Rouby Y, Combourieu E, Perrier-Gros-Claude JD, Saccharin C, Huerre M. A case of Aspergillus myocarditis associated with septic shock. J Infect 1998; 37: 295-7.

5. El-Hamamsy I, Dürrleman N, Stevens LM, Perrault LP, Carrier M. Aspergillus endocarditis after cardiac surgery. Ann Thorac Surg 2005; 80: 359-64. (Review). 